\title{
BMJ Open Study protocol for a multicentre, controlled non-randomised trial: benefits of exercise physiology services for type 2 diabetes (BEST)
}

\author{
Cecilia M Kitic, ${ }^{1}$ Steve Selig, ${ }^{2}$ Kade Davison, ${ }^{3}$ Tania L B Best, ${ }^{4}$ \\ Belinda Parmenter, ${ }^{5}$ Kate Pumpa, ${ }^{6}$ Bonnie Furzer, ${ }^{7}$ Vanessa Rice, ${ }^{8}$ \\ Sibella Hardcastle, ${ }^{1}$ Michael Cheney, ${ }^{1}$ Andrew J Palmer, ${ }^{9}$ Steve Fraser, ${ }^{2,10}$ \\ Andrew D Williams ${ }^{1}$
}

To cite: Kitic CM, Selig S, Davison $\mathrm{K}$, et al. Study protocol for a multicentre, controlled non-randomised trial: benefits of exercise physiology services for type 2 diabetes (BEST). BMJ Open 2019;9:e027610. doi:10.1136/ bmjopen-2018-027610

- Prepublication history and additional material for this paper are available online. To view these files, please visit the journal online (http://dx.doi org/10.1136/bmjopen-2018027610).

Received 01 November 2018 Revised 07 July 2019 Accepted 23 July 2019

Check for updates

(C) Author(s) (or their employer(s)) 2019. Re-use permitted under CC BY-NC. No commercial re-use. See rights and permissions. Published by BMJ.

For numbered affiliations see end of article.

Correspondence to Dr Andrew D Williams; andrew.williams@utas.edu.au

\section{ABSTRACT}

Introduction Controlled trials support the efficacy of exercise as a treatment modality for chronic conditions, yet effectiveness of real-world Exercise Physiology services is yet to be determined. This study will investigate the efficacy and cost-effectiveness of services provided by Accredited Exercise Physiologists (AEPS) for clients with type 2 diabetes (T2D) in clinical practice.

Methods and analysis A non-randomised, opportunistic control, longitudinal design trial will be conducted at ten Exercise Physiology Clinics. Participants will be individuals with T2D attending one of the Exercise Physiology Clinics for routine AEP services (exercise prescription and counselling) (intervention) or individuals with T2D not receiving AEP services (usual care) (control). The experimental period will be 6 months with measurements performed at baseline and at 6 months. Primary outcome measures will be glycosylated haemoglobin (HbA1c), resting brachial blood pressure (BP), body mass index, waist circumference, 6 min walk test, grip strength, $30 \mathrm{~s}$ sit to stand, Medical Outcomes Short-Form 36Item Health Survey and Active Australia Questionnaire. Secondary outcomes will be medication usage, out-ofpocket expenses, incidental, billable and non-billable health professional encounters and work missed through ill health. Healthcare utilisation will be measured for 12 months prior to, during and 12 months after trial participation using linked data from Medicare Benefits Schedule and Pharmaceutical Benefits Scheme data. Ethics and dissemination The study is a multicentre trial comprising: University of Tasmania, University of New South Wales Lifestyle Clinic, University of Canberra, Baker Heart and Diabetes Institute (covered under the ethics approval of University of Tasmania Health and Medical Ethics Committee H0015266), Deakin University (Approval number: 2016-187), Australian Catholic University (2016-304R), Queensland University of Technology (1600000049), University of South Australia (0000035306), University of Western Australia (RA/4/1/8282) and Canberra Hospital (ETH.8.17.170). The findings of this clinical trial will be communicated via peer-reviewed journal articles, conference presentations, social media and broadcast media.

Trial registration number ACTRN12616000264482.

\section{Strength and limitations of this study}

- The benefits of exercise physiology services for type 2 diabetes (BEST) study intervention is the delivery of routine, real-world Exercise Physiology services to manage type 2 diabetes as opposed to a clinical exercise trial.

- The study employs multiple sites across Australia to reach recruitment targets.

- There is a risk of losing participants during the 6-month intervention as Exercise Physiology services may be delivered for a duration shorter than the intervention.

- The study is a non-randomised trial due to the real-world nature of the study.

\section{INTRODUCTION}

Diabetes is estimated to affect over 422 million individuals globally, ${ }^{1}$ with type 2 diabetes (T2D) accounting for approximately $90 \%$ of diabetes incidence. ${ }^{2}$ Rising levels of physical inactivity and obesity are important contributors to the alarming increase in the incidence of T2D. ${ }^{3}$ In Australia, over $29 \%$ of adults are insufficiently active and $14 \%$ inactive (Australian Health Survey 2011-2012). In combination with its contribution to chronic disease burden, T2D is associated with a significant economic burden ${ }^{4}$ with a total annual cost in Australia of up to $\$ 6$ billion. ${ }^{5}$ With a twofold increase in the prevalence of diabetes in recent years, ${ }^{6}$ and no known cure, prevention and management strategies are key to successfully address this major public health burden.

There is substantial evidence from randomised controlled trials regarding the benefits of exercise training in diabetes management. ${ }^{7-9}$ Structured exercise training involving aerobic exercise, resistance exercises 
or a combination of both is associated with improvements in glycaemic control, ${ }^{10}$ muscle microvascular blood flow, ${ }^{11}$ muscle strength ${ }^{12}$ and quality of life ${ }^{13}{ }^{14}$ While controlled clinical studies, often conducted in hospital and university laboratory settings, demonstrate the effectiveness of exercise in the management of T2D, studies that have assessed the feasibility and outcomes of evidence-based exercise prescription in a real-world practice setting are scarce. Importantly, health benefits shown in clinical trials have not yet been proven to translate into practice where the adoption of and adherence to exercise is often more challenging. ${ }^{15}$

Primary healthcare is the main setting for T2D diagnosis, treatment and referral pathways, ${ }^{16}$ and people with T2D tend to have long-term patient:client relationships with their general practitioners ${ }^{17}$ and consider them as credible sources of health-related information. This regular and positively viewed interaction provides an opportune environment for the promotion of lifestyle medicine, and initiatives such as"'Exercise is Medicine" have been introduced to implement strategies in order to promote the effectiveness of physical activity in the healthcare setting. ${ }^{18} \mathrm{~A}$ recent meta-analysis ${ }^{19}$ reports that exercise referral in a primary healthcare setting was associated with a small increase in the adoption of physical activity (relative risk 1.12; 95\% CI 1.04 to 1.20 ). In contrast, a systematic review by Pavey $e t a l^{20}$ reports limited evidence on the clinical and cost-effectiveness of exercise referral in comparison with usual care. In the majority of the studies included in these systematic reviews, patients were referred to a non-descript exercise professional and a short-term (10-12 weeks) leisure centre-based intervention. The effectiveness of exercise referral to an exercise specialist for specific disease management is yet to be determined.

Accredited Exercise Physiologists (AEPs) are allied health professionals who have been trained to prescribe exercise and facilitate long-term behavioural change through exercise counselling in subclinical and clinical populations. While exercise prescription is the main treatment modality used by AEPs, counselling skills are also employed to facilitate behavioural change. AEPs have the professional competencies to assess clients and design and implement effective, individualised exercise interventions for people with T2D who often have significant comorbidities. Lifestyle intervention programmes are effective in reducing the progression of chronic disease $^{2122}$; however, the effects persist only as long as the new lifestyle is maintained. ${ }^{23}$

Interventions led by AEPs have been effective in increasing physical activity levels in insufficiently active adults. ${ }^{24}$ However, the only previous study that to our knowledge has examined an AEP-led exercise intervention in chronic disease evaluated the health economics associated with the intervention rather than efficacy in improving exercise/physical activity behaviour, clinical status, function and quality of life. ${ }^{25}$ The aim of this study is therefore to: (1) investigate the effectiveness of real-world Clinic AEP services for individuals with T2D on (A) sustained participation in physical activity and (B) surrogate markers of clinical prognosis including cardiovascular and metabolic risk; (2) estimate the association between different levels of session participation and surrogate markers of clinical prognosis; and (3) estimate the relative cost-effectiveness of different frequencies of attendance.

\section{METHODOLOGY}

\section{Trial design and setting}

This study comprises a non-randomised, opportunistic control, longitudinal design examining the effects of different levels of participation of individuals with T2D with Exercise Physiology services on surrogate measures of diabetes progression in real-world settings. Any amendments (if required) will be ethically approved through each site's ethics committee and communicated by the trial manager to relevant parties.

\section{Participants}

Intervention group participants will be adults ( $>18$ years) recruited from clients referred to participating clinics for Exercise Physiology services. All consecutive patients attending one of the trial centres meeting the inclusion criteria of having T2D and receiving AEP services are eligible for the intervention group. Exclusion criteria are clinically unstable patients (defined as medical factors that make exercise unsafe or patients with undiagnosed signs or symptoms suggestive of unstable chronic disease),${ }^{26}$ with contraindications to exercise or already engaged in high levels of physical activity. Participants will undergo standard AEP services, which will include: an assessment of variables associated with physical function and clinical status on their initial visit to an AEP, subsequent individualised supervised and unsupervised exercise sessions based on published exercise guidelines for individuals with $\mathrm{T} 2 \mathrm{D}^{27}$ with the dose and exact content determined by the supervising AEP in negotiation with each participant's preferences and clinical status, counselling on exercise adherence and a follow-up assessment after approximately 6 months. A control group will include participants who are not referred to Exercise Physiology services or who are on an extended waiting list for Exercise Physiology treatment and are willing to undergo an assessment of physical function and clinical status at baseline and following 6 months. This group will be recruited from clients referred for other treatments at participating clinics and from university staff and students of participating university clinics who have previously been diagnosed with T2D but have not received any AEP services. These individuals will be recruited via local flyers and social media. Participants will provide informed consent obtained from the treating AEP or trial coordinator. To avoid loss to follow-up, patients will be contacted during the follow-up period to remind them of any scheduled visits. To date, 126 of the anticipated 


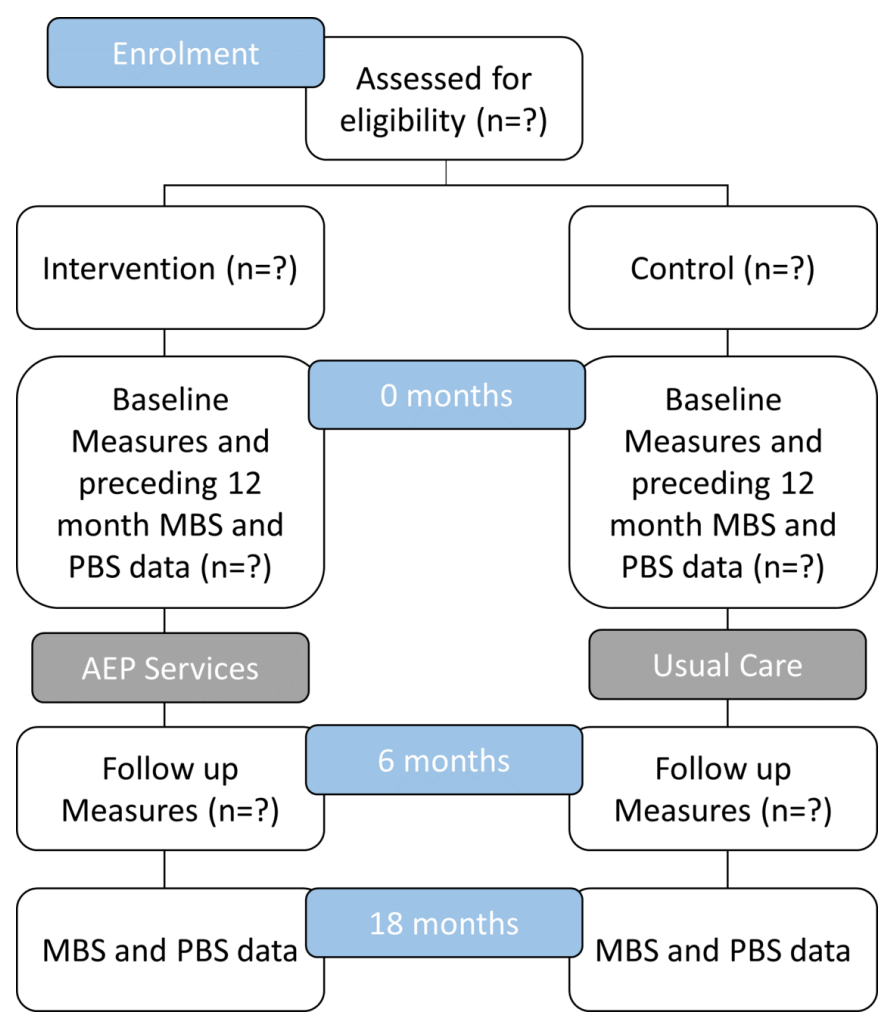

Figure 1 Flow chart of the study process. AEP, Accredited Exercise Physiologist; MBS, Medical Benefits Schedule; PBS, Pharmaceutical Benefits Scheme.

555 participants have been recruited (124 treatment and 2 controls). A flow chart of the study process is shown in figure 1. This trial protocol was developed according to the Standardized Protocol Items: Recommendations for Interventional Trials guidelines (see online supplementary file 1$)$.

\section{Data collection}

Data will be collected from all clients with T2D attending any of the participating Exercise Physiology Clinics in the trial period (April 2016-January 2021) who have provided written informed consent. Outcome data pertaining to quality of life and markers of clinical prognosis and cardiovascular risk will be collected at baseline and after 6 months. The number of sessions with an Exercise Physiologist, whether or not clients received treatment from other allied health professionals (ie, dietitian) and compliance with counselling (measured by physical function and participation in physical activity) will be recorded and used as predictor covariates in the statistical analyses. Data will be collected at each study site in custom-written Access databases (Microsoft Office 2010, Microsoft Corporation, Washington, USA). Each local database is uniquely coded for the study site. Deidentified data will be exported from the local databases and imported into a master chart for reporting and analysis. Trial data will be audited on a regular basis by the trial manager. Primary outcome measures will be glycosylated haemoglobin (HbAlc), resting brachial blood pressure (BP), body mass index (BMI), waist circumference,
6 min walk test, grip strength, $30 \mathrm{~s}$ sit to stand, Medical Outcomes Short-Form 36-Item Health Survey and Active Australia Questionnaire. Secondary outcomes will be medication usage, out-of-pocket expenses, incidental, billable and non-billable health professional encounters and work missed through ill health. Outcome data for the intervention group will be analysed against baseline data using mixed methods regression corrected for repeated measures with potential contributing variables such as age, gender, comorbidities, medications, self-reported participation, number of client AEP consultations, other allied health interventions and type of exercise prescription (eg, supervised or unsupervised and at home or in a community setting) included in the analysis as covariates. Healthcare resource utilisation will be measured for 12 months prior to enrolment, during the intervention and 12 months after trial participation for both intervention and control groups using linked data from Medicare Benefits Schedule (MBS) and Pharmaceutical Benefits Scheme (PBS) data. Medication usage, out-of-pocket expenses, incidental, billable and non-billable health professional encounters and work missed through ill health will be assessed by health diaries. The timing of study measurements is provided in table 1 .

\section{Anthropometry}

Height will be measured to the nearest $0.1 \mathrm{~cm}$ using a wall-mounted stadiometer and body weight to the nearest $0.1 \mathrm{~kg}$ using digital scales. BMI will be calculated. Waist circumference will be measured using the cross hand technique (left hand under) at the narrowest point, or at the midpoint between lowest rib and top of iliac crest if the narrowest point is not apparent. These measures are simple and easy to obtain and have been widely used as indicators of cardiovascular disease risk ${ }^{28}$ and mortality ${ }^{29}$ risk in large-scale population studies.

\section{Physical function}

The 6 min walk test (6MWT) will be used as a measure of physical function as the cardiovascular mortality risk is increased with walking distance of less than $350 \mathrm{~m}^{30}$ Briefly, participants will be instructed to walk as far as possible along a short measured course during the $6 \mathrm{~min}$. They will be permitted to stop and rest if needed and will receive standardised verbal feedback if the client stops walking or needs a rest during the test and $15 \mathrm{~s}$ prior to test completion. The $6 \mathrm{MWT}$ is a reliable and valid measure of physical function in older adults. ${ }^{31}$

\section{Musclar strength and endurance}

Strength will be measured using grip strength and muscular endurance with the 30s sit to stand (30s STS). Grip strength is a reliable and valid measure of upper body strength $^{32}$ and has been linked to outcomes, including mortality, in chronic disease. ${ }^{33}$ Grip strength will be measured with participants leaning forward slightly, with the hand to be tested in front, not touching the body; forearm will be slightly bent in a neutral position, 
Table 1 Overview of study measurements and time points for data collection

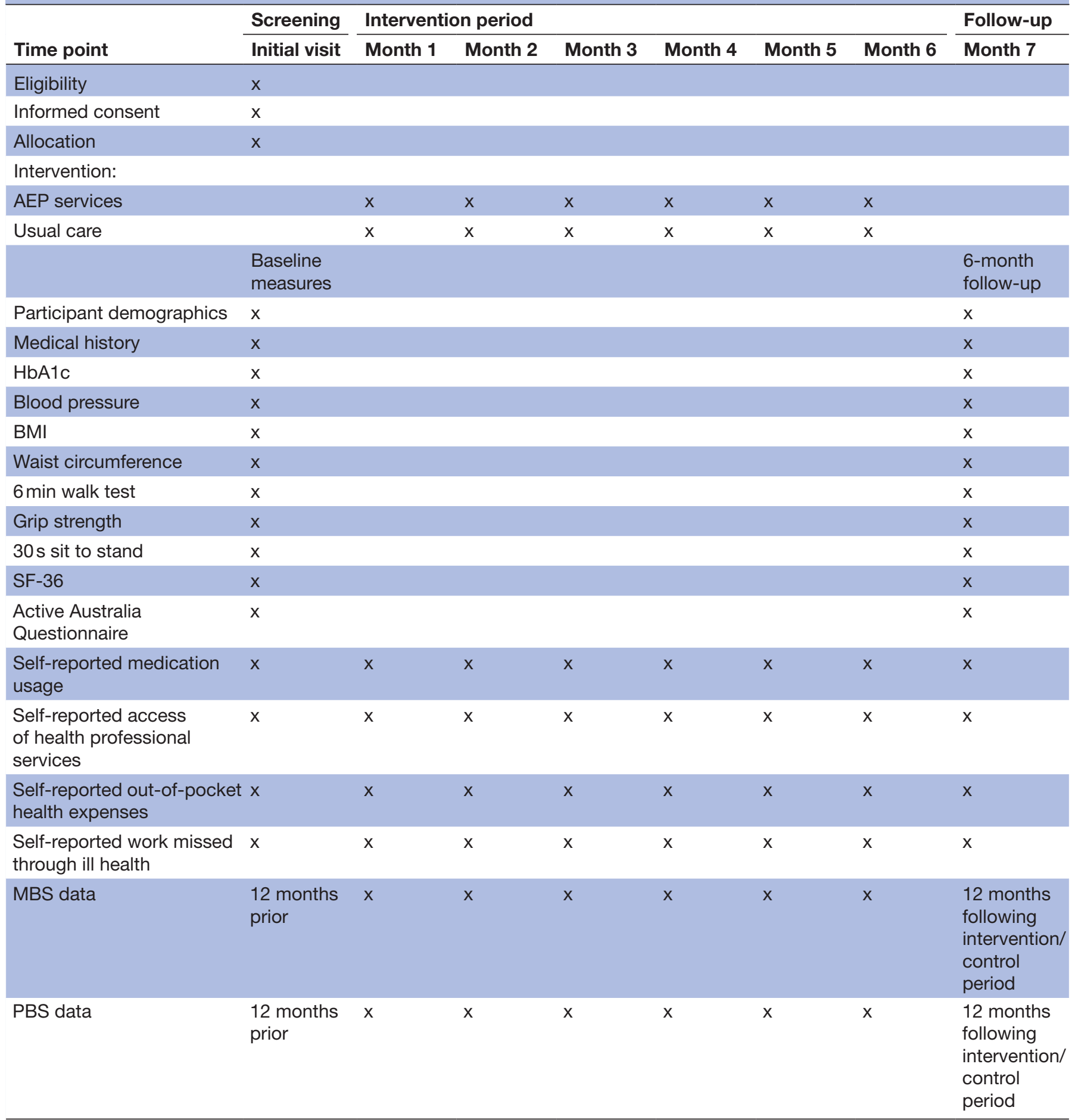

AEP, Accredited Exercise Physiologist; BMI, body mass index; HbA1c, glycosylated haemoglobin; MBS, Medicare Benefits Schedule; PBS, Pharmaceutical Benefits Scheme.

avoiding pronation or supination of the wrist as this has been shown to alter the measurement. Participants will be instructed to perform a maximum gripping effort for a few seconds while keeping the arm stationary. Two to four trials will be conducted on each arm with trials ceased on no further improvement, and the maximum scores for the right and left hands will be added together.
$30 \mathrm{~s}$ STS is a measure of lower extremity muscular endurance and function and relates to ability to perform activities of daily living. It is a reliable and valid measure that is widely used in clinical practice. ${ }^{34}$ The participant will be seated in a chair then instructed to fold their arms across their chest and to stand fully upright and sit down as many times as possible in $30 \mathrm{~s}$. 
Self-perceived physical function and health-related quality of life

Self-perceived physical function and health-related quality of life will be evaluated using the Medical Outcomes Short-Form 36-Item Health Survey (SF-36). This survey includes eight independent scales (physical functioning, role physical, bodily pain, general health, vitality, social functioning, role emotional and mental health) that are collapsed to create two global components of physical and mental dimensions of health. It is validated and has been widely used to measure quality of life in a range of chronic disease populations. ${ }^{35-37}$ The client is provided with a copy of the SF-36 and asked to answer all 36 questions as honestly as possible. The SF-36 will be divided into two summary measures: the Physical Component Summary and the Mental Component Summary.

\section{Self-reported physical activity}

Physical activity will be measured using the Active Australia questionnaire. ${ }^{38}$ Participants will be requested to include any exercise performed in the previous week in their response to the questionnaire. The survey measures frequency, intensity and duration of incidental and/or intentional physical activity in the week prior to the time of testing. The total time spent in each activity will be multiplied by an intensity value (corresponding Metabolic Equivalent; MET) and used to calculate participants' weekly physical activity in MET/min. This survey is valid and reliable ${ }^{39} 40$ and has previously been used to measure changes in physical activity in chronic disease populations. ${ }^{36}$

\section{Glycosylated haemoglobin}

Glycosylated haemoglobin (HbAlc) is a measurement of long-term glucose load in the bloodstream. Elevated concentrations of glucose in the blood react with haemoglobin in the bloodstream resulting in the haemoglobin becoming glycosylated. The concentration of HbAlc in the blood gives an indication of the glucose load over a prolonged time period ( $\sim 3$ months). As a result, this method is considered a better indicator of glucose intolerance and severity of metabolic disease than random blood glucose levels that are highly dependent on factors such as the length of time since the last meal. HbAlc is measured on a quarterly basis as part of routine diabetes management by a general practitioner. The most recent pathology results will be used to indicate HbA1c on commencement of the Exercise Physiology treatment and following 6 months.

\section{Blood pressure}

Blood pressure will be measured by sphygmomanometry using standardised techniques after 5 min of seated rest. To prevent short-term blood pressure increases, participants will be advised not to consume caffeine, exercise or smoke, 2 hours prior to assessment in line with recently published guidelines. ${ }^{41}$

\section{Health costs information}

Healthcare resource utilisation in the form of medical services received and prescribed medications received will be determined for 12 months prior to enrolment, during the intervention and 12 months after trial participation for both intervention and control groups using linked data from the MBS and PBS data. Medication usage, out-of-pocket expenses, incidental, billable and non-billable health professional encounters and work missed through ill health will be assessed by health diaries. Participants will be asked to record information in the diary on days when health services are required or when poor health prevents them from completing their usual activities. Participants will be provided with the diary and examples of how to complete the required information. Participants will detail information relating to the nature of their illness, any appointments with medical or allied health practitioners, appointment length and a brief description of what the appointment involved. Details about out-of-pocket costs (total fees charged by a medical specialist minus any Medicare rebates) associated with the illness/appointment will also be recorded (this may include the cost of the appointment itself, medications or equipment).

\section{Cost-effectiveness analysis}

Incremental cost-effectiveness ratios will be calculated as the difference in direct and indirect costs between the two groups, divided by the incremental quality-adjusted life years gained (derived from SF-36-derived health utilities).

\section{Reproducibility of study procedures}

A manual of standard operations and procedures has been developed to standardise all testing procedures and Exercise Physiologist training in areas including patient recruitment, measurement, assessment, data entry, security and management to assist reliability and accuracy of measures obtained across all participating sites.

\section{Data analysis}

Sample size was calculated on the basis of participant numbers required to achieve statistically and clinically significant differences in HbAlc (alpha $=0.05$ ) on both between group (intervention vs control) and within group (intervention participants divided into two groups on the basis of exercise compliance) analyses. ${ }^{42}$ The calculation revealed that 99 participants will be required per group to detect a change in HbA1c of $0.4 \%$, SD 1.0 , power $80 \%$. As potential difficulties are expected in accessing patients with T2D who have not received a referral for Exercise Physiology services, as well as budgetary constraints, we have performed a sample size calculation for unequal groups (http://epitools.ausvet.com.au/content.php? page $=2$ Means2). This calculation indicated that we require 500 individuals in the intervention group and 55 individuals in the control group. Consequently, 500 individuals with T2D who have been referred for an Exercise Physiology intervention will be recruited for 
the intervention, while 55 patients with T2D who are not receiving Exercise Physiology services will be recruited for the control group by opportunistic sample.

Intervention versus control group data will be assessed for changes in health-related variables and behaviours over time via mixed methods linear regression. Where assumptions of linear regression are violated, data will be analysed using non-parametric analysis via ordinal logistic regression. Outcome data for the intervention group will be analysed against baseline data using adjusted and unadjusted mixed methods regression with potential contributing variables such as age, gender, comorbidities, medications, self-reported participation, number of client Exercise Physiology consultations, other allied health interventions and types of exercise prescription (home based or community setting) considered in the modelling as potential covariates. The internal cost-effectiveness of the programme (the relationship between rising exercise clinic costs as treatment frequency increases against improving exercise compliance with counselling) will also be estimated. Analyses will be performed using STATA SE 15.0 with statistical significance set at $\mathrm{p}<0.05$.

\section{Patient and public involvement}

Patients or the public were not directly involved in the design of this study. The study is designed to quantify the effectiveness of Exercise Physiology services that are routinely delivered in a best-practice, patient-centred manner.

\section{DISCUSSION}

Effective management strategies are required to address the increasing global epidemic of T2D. Controlled trials support the efficacy of exercise as a treatment modality, ${ }^{7-9}$ and clinical trials have demonstrated the effectiveness of Exercise Physiologists to influence long-term behavioural change in insufficiently active adults. ${ }^{24}$ Additionally, a commissioned report in 2015 on the value of Accredited Exercise Physiologists in Australia suggested health system cost savings of up to $\$ 5107$ per person (cost benefit ratio of 8.8 to 1) for individuals with T2D accessing Exercise Physiology services, ${ }^{43}$ under the assumption that efficacy from clinical trials translated to real-world Exercise Physiology services. On the basis of assumptions that Exercise Physiology services are effective, an individual with T2D in Australia may access up to eight group and five individual Exercise Physiology services in a calendar year following an appropriate referral from their general practitioner. Despite this, the effectiveness of real-world Exercise Physiology services in Australia is yet to be determined.

This study is unique as it aims to bridge an important gap between controlled, clinical trials and real-world practice, where the effectiveness of the translation of this evidence base to health improvements requires quantification. The work will quantify the health and economic outcomes of Exercise Physiology services for T2D in a real-world setting, providing valuable data for facilitating the management strategies of T2D and also may help in developing health policies for T2D patients.

\section{ETHICS AND DISSEMINATION}

The study will be conducted in accordance with the National Health and Medical Research Council national statement on ethical conduct in research, as well as the approved study protocol. A detailed participant information sheet will be provided to each individual prior to them providing written, informed consent.

The findings of this clinical trial will be communicated using a comprehensive dissemination strategy that includes peer-reviewed journal articles, conference presentations, social media and broadcast media. No professional writers are intended to be used in the preparation of associated presentations, manuscripts and materials, and author eligibility will be determined using the International Committee of Medical Journal Editors authorship guide.

\section{Author affiliations}

${ }^{1}$ School of Health Sciences, University of Tasmania, Launceston, Tasmania, Australia ${ }^{2}$ School of Exercise and Nutrition Sciences, Deakin University, Melbourne, Victoria, Australia

${ }^{3}$ School of Health Sciences, Univesity of South Australia, Adelaide, South Australia, Australia

${ }^{4}$ School of Exercise and Nutrition Sciences, Queensland University of Technology, Brisbane, Queensland, Australia

${ }^{5}$ Department of Exercise Physiology, University of New South Wales, Sydney, New South Wales, Australia

${ }^{6}$ Faculty of Health, University of Canberra, Canberra, Australian Capital Territory, Australia

${ }^{7}$ School of Health Sciences, University of Western Australia, Perth, Western Australia, Australia

${ }^{8}$ Faculty of Health Science, Australian Catholic University, Melbourne, Queensland, Australia

${ }^{9}$ Menzies Institute for Medical Research, University of Tasmania, Hobart, Tasmania, Australia

${ }^{10}$ Institute for Physical Activity and Nutrition, Deakin University, Melbourne, Victoria, Australia

Acknowledgements ADW is supported by a Medical Research Future Fund TRIP Fellowship.

Contributors ADW, SS, KD, TLBB, CMK, BP, KP, BF, VR and SH were investigators of the successful funding proposal. MC will act as trial coordinator. CMK and ADW wrote the manuscript, while BF, KP, BP, AJP, SF, SH and TLBB reviewed the manuscript. All authors have read and approved the final version.

Funding This work is in part supported by Exercise \& Sports Science Australia. Competing interests None declared.

Patient consent for publication Not required.

Ethics approval This study has been approved by the following ethics committees: University of Tasmania, University of New South Wales Lifestyle Clinic, University of Canberra, Baker Heart and Diabetes Institute are all covered under the approval of the University of Tasmania Health and Medical Ethics Committee H0015266, Deakin University (Approval number: 2016-187), Australian Catholic University (2016304R), Queensland University of Technology (1600000049), University of South Australia (0000035306), University of Western Australia (RA/4/1/8282) and the Canberra Hospital (ETH.8.17.170). The trial has been registered with the Australian New Zealand Clinical Trials Registry ACTRN12616000264482.

Provenance and peer review Not commissioned; externally peer reviewed.

Open access This is an open access article distributed in accordance with the Creative Commons Attribution Non Commercial (CC BY-NC 4.0) license, which 
permits others to distribute, remix, adapt, build upon this work non-commercially, and license their derivative works on different terms, provided the original work is properly cited, appropriate credit is given, any changes made indicated, and the use is non-commercial. See: http://creativecommons.org/licenses/by-nc/4.0/.

\section{REFERENCES}

1. Zhou B, Lu Y, Hajifathalian K, et al. Worldwide trends in diabetes since 1980: a pooled analysis of 751 population-based studies with 4.4 million participants. Lancet 2016;387:1513-30.

2. World Health Organisation. Definition, diagnosis and classification of diabetes mellitus and its complications. Part 1: diagnosis and classification of diabetes mellitus. Geneva: World Health Organisation, 1999.

3. Venables MC, Jeukendrup AE. Physical inactivity and obesity: links with insulin resistance and type 2 diabetes mellitus. Diabetes Metab Res Rev 2009;25(Suppl 1):S18-23.

4. Seuring T, Archangelidi $O$, Suhrcke M. The economic costs of type 2 diabetes: a global systematic review. Pharmacoeconomics 2015;33:811-31.

5. Shaw J, Tanamas S. Diabetes: the silent pandemic and its impact on Australia baker IDI heart and diabetes Institute, 2012.

6. Mathers CD, Loncar D. Projections of global mortality and burden of disease from 2002 to 2030. PLoS Med 2006;3:e442.

7. Villafaina S, Collado-Mateo D, Fuentes JP, et al. Physical exercise improves heart rate variability in patients with type 2 diabetes: a systematic review. Curr Diab Rep 2017;17:110.

8. Pai L-W, Chang P-Y, Chen W, et al. The effectiveness of physical leisure time activities on glycaemic control in adult patients with diabetes type 2: a systematic review. JBI Libr Syst Rev 2012;10(42 Suppl):1-20.

9. Praet SFE, van Loon LJC. Optimizing the therapeutic benefits of exercise in type 2 diabetes. J Appl Physiol 2007;103:1113-20.

10. Umpierre D, Ribeiro PAB, Kramer CK, et al. Physical activity advice only or structured exercise training and association with $\mathrm{HbA1c}$ levels in type 2 diabetes: a systematic review and meta-analysis. JAMA 2011:305:1790-9. 10

11. Russell RD, Hu D, Greenaway T, et al. Skeletal muscle MicrovascularLinked improvements in glycemic control from resistance training in individuals with type 2 diabetes. Diabetes Care 2017;40:1256-63.

12. Lee J, Kim D, Kim C. Resistance training for glycemic control, muscular strength, and lean body mass in old type 2 diabetic patients: a meta-analysis. Diabetes Ther 2017;8:459-73.

13. SD R, Schuch FB, Kanitz AC, et al. Quality of life and sleep quality are similarly improved after aquatic or dry-land aerobic training in patients with type 2 diabetes: a randomized clinical trial. J Sci Med Sport 2017.

14. Grace A, Chan E, Giallauria F, et al. Clinical outcomes and glycaemic responses to different aerobic exercise training intensities in type II diabetes: a systematic review and meta-analysis. Cardiovasc Diabetol 2017;16:37

15. Advika TS, Idiculla J, Kumari SJ. Exercise in patients with Type 2 diabetes: Facilitators and barriers - A qualitative study. J Family Med Prim Care 2017;6:288-92.

16. Royal Australian College of General Practitioners, Diabetes Australia. Diabetes management in general practice: guidelines for type 2 diabetes. 7th ed, 2011

17. Lethbridge-Cejku M, Schiller JS, Bernadel L. Summary health statistics for U.S. adults: National health interview survey, 2002. Vital Health Stat 2004;10:1-151.

18. Lobelo F, Stoutenberg M, Hutber A. The exercise is medicine global health Initiative: a 2014 update. Br J Sports Med 2014;48:1627-33.

19. Campbell F, Holmes M, Everson-Hock E, et al. A systematic review and economic evaluation of exercise referral schemes in primary care: a short report. Health Technol Assess 2015;19:1-110.

20. Pavey TG, Taylor AH, Fox KR, et al. Effect of exercise referra schemes in primary care on physical activity and improving health outcomes: systematic review and meta-analysis. BMJ 2011;343:d6462.

21. Hambrecht R, Walther C, Möbius-Winkler S, et al. Percutaneous coronary angioplasty compared with exercise training in patients with stable coronary artery disease: a randomized trial. Circulation 2004;109:1371-8.

22. Herman $\mathrm{WH}$, Hoerger $\mathrm{TJ}$, Brandle $\mathrm{M}$, et al. The cost-effectiveness of lifestyle modification or metformin in preventing type 2 diabetes in adults with impaired glucose tolerance. Ann Intern Med 2005;142:323-32.

23. Lindahl B, Nilssön TK, Borch-Johnsen $\mathrm{K}$, et al. A randomized lifestyle intervention with 5-year follow-up in subjects with impaired glucose tolerance: pronounced short-term impact but long-term adherence problems. Scand J Public Health 2009;37:434-42.

24. Ewald B, Stacey F, Johnson N, et al. Physical activity coaching by Australian exercise Physiologists is cost effective for patients referred from general practice. Aust N Z J Public Health 2018;42:12-15.

25. Bertram MY, Lim SS, Barendregt JJ, et al. Assessing the costeffectiveness of drug and lifestyle intervention following opportunistic screening for pre-diabetes in primary care. Diabetologia 2010;53:875-81.

26. Maiorana AJ, Williams AD, Askew CD, et al. Exercise professionals with advanced clinical training should be afforded greater responsibility in Pre-Participation exercise screening: a new collaborative model between exercise professionals and physicians. Sports Med 2018;48:1293-302.

27. Hordern MD, Dunstan DW, Prins JB, et al. Exercise prescription for patients with type 2 diabetes and pre-diabetes: a position statement from exercise and sport science Australia. J Sci Med Sport 2012;15:25-31

28. Dis van I, Kromhout D, Geleijnse JM, et al. Body mass index and waist circumference predict both 10-year nonfatal and fatal cardiovascular disease risk: study conducted in 20,000 Dutch men and women aged 20-65 years. Eur J Cardiovasc Prev Rehabil 2009;16:729-34.

29. Sui X, LaMonte MJ, Laditka JN, et al. Cardiorespiratory fitness and adiposity as mortality predictors in older adults. JAMA 2007;298:2507-16

30. Rasekaba T, Lee AL, Naughton MT, et al. The six-minute walk test: a useful metric for the cardiopulmonary patient. Intern Med J 2009;39:495-501.

31. Harada ND, Chiu V, Stewart AL. Mobility-related function in older adults: assessment with a 6-minute walk test. Arch Phys Med Rehabil 1999;80:837-41.

32. Wang C-Y, Chen L-Y. Grip strength in older adults: test-retest reliability and cutoff for subjective weakness of using the hands in heavy tasks. Arch Phys Med Rehabil 2010;91:1747-51.

33. Sasaki H, Kasagi F, Yamada M, et al. Grip strength predicts causespecific mortality in middle-aged and elderly persons. Am J Med 2007;120:337-42.

34. Jones CJ, Rikli RE, Beam WC. A 30-S Chair-Stand test as a measure of lower body strength in Community-Residing older adults. Res $Q$ Exerc Sport 1999;70:113-9.

35. Esteban C, Quintana JM, Aburto M, et al. Impact of changes in physical activity on health-related quality of life among patients with COPD. Eur Respir J 2010;36:292-300.

36. Koh KP, Fassett RG, Sharman JE, et al. Effect of intradialytic versus home-based aerobic exercise training on physical function and vascular parameters in hemodialysis patients: a randomized pilot study. Am J Kidney Dis 2010;55:88-99.

37. Reid RD, Tulloch HE, Sigal RJ, et al. Effects of aerobic exercise, resistance exercise or both, on patient-reported health status and well-being in type 2 diabetes mellitus: a randomised trial. Diabetologia 2009 (published Online First: 2009/12/17).

38. Australian Institute of Health and Welfare. The active Australia survey: a guide and manual for implementation, analysis and reporting. Canberra: AlHW, 2003.

39. Brown WJ, Burton NW, Marshall AL, et al. Reliability and validity of a modified self-administered version of the active Australia physical activity survey in a sample of mid-age women. Aust N Z J Public Health 2008;32:535-41.

40. Pettee Gabriel K, McClain JJ, Lee CD, et al. Evaluation of physical activity measures used in middle-aged women. Med Sci Sports Exerc 2009;41:1403-12.

41. Whelton PK, Carey RM, Aronow WS, et al. 2017 ACC/AHA/AAPA ABC/ACPM/AGS/APhA/ASH/ASPC/NMA/PCNA guideline for the prevention, detection, evaluation, and management of high blood pressure in adults: a report of the American College of Cardiology/ American heart association Task force on clinical practice guidelines. Hypertension 2018;71:e13-115.

42. Selvin E, Crainiceanu CM, Brancati FL, et al. Short-Term variability in measures of glycemia and implications for the classification of diabetes. Arch Intern Med 2007;167:1545-51.

43. Deloitte Access Economics. Value of accredited exercise Physiologists in Australia, 2015. https://www2.deloitte.com/au/en/ pages/economics/articles/value-exercise-physiologists-australia.html 